5. J. Milnor, Morse theory, Annals of Mathematics Studies, No. 51, Princeton Univ. Press, Princeton, N. J., 1963.

6. F. P. Peterson, Some remarks on Chern classes, Ann. of Math. 69 (1959), 414-420.

7. K. J. Ramspott, Stetige und holomorphe Schnitte in Bündeln mit homogener Faser, Math. Z. 89 (1965), 234-246.

8. E. Stiefel, Richtungsfelder und Fernparallelismus in n-dimensionalen Mannigfaltigkeiten, Comment. Math. Helv. 8 (1935), 305-353.

Institute for AdVANCED StUdy

\title{
AUTOMORPHISM GROUPS OF FINITELY GENERATED NILPOTENT GROUPS
}

\author{
BY LOUIS AUSLANDER AND GILBERT BAUMSLAG ${ }^{1}$
}

Communicated by David A. Buchsbaum, April 5, 1967

It is rare for any property of a group $G$ to carry over to its automorphism group. Recently J. Lewin [1] constructed a finitely presented group whose automorphism group is not even finitely generated. Now finitely generated nilpotent groups are finitely presented (see e.g. [2]). So Lewin's example contrasts strikingly with the following.

THEOREM A. The automorphism group of a finitely generated nilpotent group is finitely presented.

In a way Theorem $A$ reinforces the commonly held view that the automorphism group of a finitely generated nilpotent group is, from a group-theoretical viewpoint, quite simple. Now Philip Hall [3] has proved that a finitely generated nilpotent group has a faithful representation in $\mathrm{GL}(n, Z)$, the integer unimodular group of degree $n$. So the following generalization of Hall's theorem might be thought of as another indication of the controlled nature of finitely generated nilpotent groups and their automorphism groups.

THEOREM B. The holomorph of a finitely generated nilpotent group (i.e. the split extension of the group by its automorphism group) has a faithful representation in $\mathrm{GL}(n, Z)$ for some $n$.

The proofs of Theorem A and Theorem B use general Lie-theoretic techniques and a result which is of independent interest, namely

${ }^{1}$ Help from the N.S.F. is gratefully acknowledged. The second author holds a Sloan Fellowship. 
Theorem $\mathrm{C}$ below. In order to state Theorem $\mathrm{C}$ we recall that two groups are commensurable if they are both finite extensions of a common group.

THEOREM C. The automorphism groups of commensurable finitely generated nilpotent groups are commensurable.

It seems likely that these theorems have analogues for polycyclic groups. These analogues and complete proofs of Theorems A, B, and $\mathrm{C}$ will appear elsewhere.

\section{REFERENCES}

1. J. Lewin, Ph.D. thesis, New York University, New York, 1964.

2. P. Hall, Finiteness condutions for solvable groups, Proc. London Math. Soc. (3) 4 (1954), 419-436.

3. - Nilpotent groups, Canadian Mathematical Congress (1957), University of Alberta, Canada.

City University of New York 